\title{
Discrete variational integrators and optimal control theory
}

\author{
Manuel DE LEÓN * David MARTÍN DE DIEGO ${ }^{\dagger}$ \\ Aitor SANTAMARÍA-MERINO \\ Laboratory of Dynamical Systems, Mechanics and Control \\ Instituto de Matemáticas y Física Fundamental \\ Consejo Superior de Investigaciones Científicas \\ Serrano 123, 28006 Madrid, SPAIN
}

December 10, 2007

\begin{abstract}
A geometric derivation of numerical integrators for optimal control problems is proposed. It is based in the classical technique of generating functions adapted to the special features of optimal control problems.
\end{abstract}

\section{Introduction}

Optimal control has been one of the driving forces behind many of the applications of mathematics to engineering, robotics, economics... In fact, the Maximum Principle was discovered by L.S. Pontryagin in 1955 in an attempt to find a solution for a highly specific optimization problem related to the manoeuvres of an aircraft. One of its main features is the interplay among different research areas, specially control theory, classical mechanics and differential geometry. Historically, Optimal Control Theory (OCT) took place during the 1950's and its geometrization was started in the 1960's. This geometric analysis of OCT has been introduced using many fundamental tools of differential geometry: Lie groups, exterior differential systems, fiber bundles, riemannian and subriemannian geometry among others.

From other point of view, a geometric methodology has been recently shown to be very useful for simulating numerically the motion of dynamical systems. Following

*mdeleon@imaff.cfmac.csic.es

$\dagger$ d.martin@imaff.cfmac.csic.es

†aitors@imaff.cfmac.csic.es 
this research line, new numerical methods have been developed, called geometric integrators; usually, these integrators, in simulations, can run for longer times with lower spurious effects (for instance, bad energy behavior for conservative systems) than the traditional (non-geometrical) ones. In particular, we are interested in extensions to OCT of Discrete variational integrators. These integrators have precisely their roots in the optimal control literature in the 1960's and 1970's (Jordan and Polack [JorPol:64, Cadzow Cadz:70, Maeda Mae:80, Mae:81]) and in 1980's by Lee [Lee:83, Lee:87, Moser and Veselov MosVes:91. Although this kind of symplectic integrators have been considered for conservative systems JarNor:97a, KaMaOr:99, MarWes:01, it has been recently shown how discrete variational mechanics can include forced or dissipative systems KMOW:00, MarWes:01, holonomic constraints MarWes:01, time-dependent systems LeoMdD:2002, MarWes:01, frictional contact PKMO:02 and nonholonomic constraints (see Cort:02, CorMar:01, LeMDSa:02a, LeMDSa:02b]). Moreover, it has been also discussed reduction theory BobSus:99a, BobSus:99b, extension to field theories JarNor:97b, MaPaSh:98, and quantum mechanics [NorJar:98].

In this paper, we shall continue this work by extending to the discrete variational techniques to Optimal Control Problems and relating our results with Discrete Optimal Control Theory. Mainly, we shall give a geometrical construction of symplectic integrators for OCT, proving as a direct consequence the symplecticity of some discrete optimal control problems. As a nice consequence, an easy proof of the symplecticity of discrete Hamilton equations will be given.

Since most engineering systems are time-dependent, we shall include the time variable explicitely in our control models and some geometric tools (mainly, cosymplectic geometry) of time-dependent mechanics will be useful

\section{Optimal control theory}

It is well known that the dynamics of a large class of engineering and economic systems can be expressed as a set of differential equations

$$
\dot{q}^{A}=\Gamma^{A}(t, q(t), u(t)), 1 \leq A \leq n,
$$

where $t$ is the time, $q^{A}$ denote the state variables and $u^{a}, 1 \leq a \leq m$, the control inputs to the system that must be specified. Given an initial condition of the state variables and given control inputs we completely know the trajectory of the state variables $q(t)$ (all the functions are assumed to be at least $C^{2}$ ).

Given an initial condition, usually $q_{0}=q\left(t_{0}\right)$, our aim is to find a $C^{2}$-piecewise smooth curve $\gamma(t)=(q(t), u(t))$, satisfying the control equations (11) and minimizing the functional

$$
\mathcal{J}(\gamma)=\int_{t_{0}}^{T} L(t, q(t), u(t)) d t+S(T, q(T)),
$$

for some fixed and given final time $T \in \mathbb{R}^{+}$. The integral $\int_{t_{0}}^{T} L(t, q(t), u(t)) d t$ depends on the time history (from $t_{0}$ to $T$ ) of the state variables and the control inputs, and $S(\cdot, q(\cdot))$ is a cost function based on the final time and the final states of the system.

In a global description, one assumes a fiber bundle structure $\pi: \mathbb{R} \times C \longrightarrow Q$, where $Q$ is the configuration manifold with local coordinates $\left(q^{A}\right)$ and $C$ is the bundle of controls, with coordinates $\left(q^{A}, u^{a}\right), 1 \leq A \leq n, 1 \leq a \leq m$. 
The time-dependent ordinary differential equations (11) on $Q$ depending on the parameters $u$ can be seen as a vector field $\Gamma$ along the projection map $\pi$, that is, $\Gamma$ is a smooth map $\Gamma: \mathbb{R} \times C \longrightarrow T Q$ such that the diagram

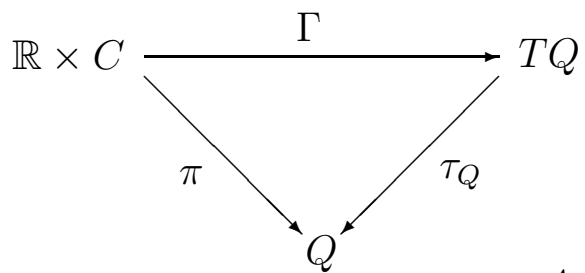

is commutative. This vector field is locally written as $\Gamma=\Gamma^{A}(t, q, u) \frac{\partial}{\partial q^{A}}$.

A neccesary condition for the solutions of such problem are provided by Pontryaguin's maximum principle. If we construct the pseudo-Hamiltonian function:

$$
H(t, q, p, u)=p_{A} \Gamma^{A}(t, q, u)-L(t, q, u)=p \Gamma(t, q, u)-L(t, q, u)
$$

where $p_{A}, 1 \leq A \leq n$, are now considered as Lagrange's multipliers, then a curve $\gamma:\left[t_{0}, T\right] \rightarrow C, \gamma(t)=(q(t), u(t))$ is an optimal trajectory if there exist functions $p_{A}(t), 1 \leq A \leq n$, such that they are solutions of the pseudo-Hamilton equations:

$$
\left\{\begin{array}{l}
\dot{q}^{A}(t)=\frac{\partial H}{\partial p_{A}}(t, q(t), p(t), u(t)) \\
\dot{p}_{A}(t)=-\frac{\partial H}{\partial q^{A}}(t, q(t), p(t), u(t))
\end{array}\right.
$$

and we have

$$
H(t, q(t), p(t), u(t))=\min _{v} H(t, q(t), p(t), v), \quad t \in\left[t_{0}, T\right]
$$

with transversality conditions

$$
q(0)=q_{0} \quad \text { and } \quad p_{A}(T)=-\frac{\partial S}{\partial q^{A}}(T, x(T))
$$

Condition (5) is usually replaced by

$$
\frac{\partial H}{\partial u^{a}}=0, \quad 1 \leq a \leq m,
$$

when we are looking for extremal trajectories.

It is well known that the Pontryaguin's necessary conditions for extremality have a geometric interpretation in terms of presymplectic (or precosymplectic) Hamiltonian systems. The total space of the system will be $\mathbb{R} \times\left(T^{*} Q \times{ }_{Q} C\right)$, with induced coordinates $\left(t, q^{A}, p_{A}, u^{a}\right)$.

Define the Pontryaguin's Hamiltonian function $H: \mathbb{R} \times\left(T^{*} Q \times{ }_{Q} C\right) \longrightarrow \mathbb{R}$ as follows

$$
H\left(t, \alpha_{q}, u_{q}\right)=\left\langle\alpha_{q}, \Gamma\left(t, u_{q}\right)\right\rangle-L\left(t, u_{q}\right)
$$

where $\alpha_{q} \in T_{q}^{*} Q$ and $\left(t, u_{q}\right) \in \pi^{-1}(q)$. Therefore, the coordinate expression of $H$ is (3).

Let $\omega_{Q}=-d \theta_{Q}$ be the canonical symplectic form on $T^{*} Q$, where $\theta_{Q}$ is the Liouville form, and consider the canonical projection $\pi_{1}: \mathbb{R} \times\left(T^{*} Q \times{ }_{Q} C\right) \longrightarrow T^{*} Q$. Define 
the 2 -form $\Omega_{H}$ on $\mathbb{R} \times\left(T^{*} Q \times{ }_{Q} C\right)$ by $\Omega_{H}=\pi_{1}^{*} \omega_{Q}+d H \wedge d t$. Then, $\left(d t, \Omega_{H}\right)$ is a precosymplectic structure on $\mathbb{R} \times\left(T^{*} Q \times{ }_{Q} C\right)$ (see [LeMaMD:96]).

Eqs. (44) and (6) can be intrinsically written as

$$
i_{X} \Omega_{H}=0, \quad i_{X} d t=1
$$

Since $\left(d t, \Omega_{H}\right)$ is a precosymplectic structure, Eqs. (7) need not have a solution, in general.

Applying the Dirac-Bergmann-Gotay-Nester algorithmn [Dirac:64, GotNes:79] to the precosymplectic system

$$
\left(\mathbb{R} \times\left(T^{*} Q \times{ }_{Q} C\right), d t, \Omega_{H}, H\right)
$$

(see [ChLeMa:94]) we obtain that Eqs. (6) correspond to the primary constraints for the precosymplectic system:

$$
\phi^{a}=\frac{\partial H}{\partial u^{a}}=0
$$

Eqs. (77) have algebraic solution along the first constraint submanifold $P_{0}$ determined by the vanishing of the primary constraints. On the points of $P_{0}$ there is at least a pointwise solution of Eq. (7), but such solutions are not, in general, tangent to $P_{0}$. These points must be removed leaving a subset $P_{1} \subset P_{0}$ (it is assumed than $P_{1}$ also is a submanifold). Thus, we have to restrict to a submanifold $P_{2}$ where the solutions of (7) are tangent to $P_{1}$. Proceeding further this way, we obtain a sequence of submanifolds

$$
\cdots \hookrightarrow P_{k} \hookrightarrow \cdots \hookrightarrow P_{2} \hookrightarrow P_{1} \hookrightarrow P_{0} \hookrightarrow \mathbb{R} \times\left(T^{*} Q \times{ }_{Q} C\right)
$$

If this algorithm stabilizes, i.e. there exists a positive integer $k \in \mathbb{N}$ such that $P_{k}=$ $P_{k+1}$ and $\operatorname{dim} P_{k} \neq 0$, then we shall obtain a final submanifold $P_{f}=P_{k}$, on which a vector field $X$ exists such that

$$
\left(i_{X} \Omega_{H}\right)_{\mid P_{f}}=0, \quad\left(i_{X} d t=1\right)_{\mid P_{f}}
$$

The constraints determining $P_{f}$ are known, in the control literature, as higher order conditions for optimality.

If $X$ is a solution of (8) then every arbitrary solution on $P_{f}$ is of the form $X^{\prime}=X+\xi$, where $\xi \in\left(\operatorname{ker} \Omega_{H} \cap \operatorname{ker} d t\right) \cap T P_{f}$.

Therefore, a necessary condition for optimality of the curve $\gamma: \mathbb{R} \rightarrow \mathbb{R} \times C$, $\gamma(t)=(t, q(t), u(t))$ is the existence of a lift $\tilde{\gamma}$ of $\gamma$ to $P_{f}$ such that $\tilde{\gamma}$ is an integral curve of a solution to Eqs. (8) .

In the regular case, the final constraint manifold will be $P_{0}$ (that is, $P_{0}=P_{f}$ ) and all the constraints are of the second kind following the classification of Dirac (see LeMaMD:96]). In such case, $\left(P_{0}, \Omega, \eta\right)$ is a cosymplectic manifold, where $\Omega$ and $\eta$ denote the restrictions of $\Omega_{H}$ and $d t$ to the submanifold $P_{0}$. Denote also by $\omega$ and $\theta$ the restrictions of $\pi_{1}^{*} \omega_{Q}$ and $\pi_{1}^{*} \theta_{Q}$ to $P_{0}$.

The cosymplecticity of $\left(P_{0}, \eta, \Omega\right)$ is locally equivalent to the regularity of the matrix

$$
\left(\frac{\partial^{2} H}{\partial u^{a} \partial u^{b}}\right)_{1 \leq a, b \leq m}
$$

along $P_{0}$. The dynamical equations for the optimal control problem will become

$$
i_{X} \Omega=0, \quad i_{X} \eta=1
$$


Taking coordinates $\left(t, q^{A}, p_{A}\right)$ on $P_{0}$, then (9) are equivalent to:

$$
\left\{\begin{array}{l}
\dot{q}^{A}(t)=\frac{\partial H_{\mid P_{0}}}{\partial p_{A}}(t, q(t), p(t)) \\
\dot{p}_{A}(t)=-\frac{\partial H_{\mid P_{0}}}{\partial q^{A}}(t, q(t), p(t)),
\end{array}\right.
$$

where we have substituted in (44) the control variables $u^{a}$ by its value $\bar{u}^{a}=f^{a}(t, q, p)$, applying the Implicit Function Theorem to the primary constraints $\phi^{a}=0$. This also implies that we have a canonical projection from $P_{0}$ onto $\mathbb{R}$, say $\pi_{0}: P_{0} \rightarrow \mathbb{R}$.

In such case, there exists a unique solution $X_{P_{0}}$ of Eq. (9):

$$
i_{X_{P_{0}}} \Omega=0, \quad i_{X_{P_{0}}} \eta=1
$$

and its flow preserves the cosymplectic structure given by $\Omega$ and $\eta$. That is, if we denote by $F_{h}$ the flow of $X_{P_{0}}$ then $F_{h}^{*} \Omega=\Omega$ and $F_{h}^{*} \eta=\eta$. In local coordinates, $F_{h}\left(t_{0}, q_{0}, p_{0}\right)=\left(t_{0}+h, q_{1}, p_{1}\right)$. Denote by $F_{h}^{(2)}$ the mapping $F_{h}^{(2)}\left(t_{0}, q_{0}, p_{0}\right)=\left(q_{1}, p_{1}\right)$, and by $F_{t_{1}, t_{0}}: P_{0}^{t_{0}} \longrightarrow P_{0}^{t_{1}}$ the mapping defined by

$$
F_{t_{1}, t_{0}}\left(q_{0}, p_{0}\right)=F_{t_{1}-t_{0}}^{(2)}\left(t_{0}, q_{0}, p_{0}\right)
$$

where we write $P_{0}^{t}=\left(\pi_{0}\right)^{-1}(t)$, with $t \in \mathbb{R}$. Obviously, $F_{t_{2}, t_{1}} \circ F_{t_{1}, t_{0}}=F_{t_{2}, t_{0}}$ in their common domain.

The submanifolds $P_{0}^{t}$ naturally inherit a symplectic structure $\omega_{t}$ by taking the restriction of $\omega$ to $P_{0}^{t}$. Similarly, denote by $\theta_{t}$ the restriction of $\theta$ to $P_{0}^{t}$, then $\omega_{t}=-d \theta_{t}$.

It is easy to deduce that, in such case, $F_{t_{1}, t_{0}}$ is a symplectomorphism; that is, $F_{t_{1}, t_{0}}^{*} \omega_{t_{1}}=\omega_{t_{0}}$, noting that

$$
\Omega=\omega+d H_{\mid P_{0}} \wedge \eta
$$

This last remark will be interesting for constructing geometrical integrators for explicitly time-dependent optimal control systems.

\section{Generating functions}

Let $\left(M_{i}, \omega_{i}\right), i=0,1$ be two exact symplectic manifolds (i.e. $\omega_{i}$ is symplectic and exact, $\left.\omega_{i}=-d \theta_{i}, i=0,1\right)$ and suppose that $g: M_{0} \rightarrow M_{1}$ is a diffeomorphism. Denote by $\operatorname{Graph}(g)$ the graph of $g, \operatorname{Graph}(g)=\left\{\left(x_{0}, g\left(x_{0}\right)\right) / x_{0} \in M_{0}\right\} \subset M_{0} \times M_{1}$. Denote by $\pi_{i}: M_{0} \times M_{1} \rightarrow M_{i}, i=0,1$ the canonical projections, and consider the 1-form and 2-form on $M_{0} \times M_{1}$ defined by

$$
\begin{aligned}
& \Theta_{(1,0)}=\pi_{1}^{*} \theta_{1}-\pi_{0}^{*} \theta_{0} \\
& \Omega_{(1,0)}=\pi_{1}^{*} \omega_{1}-\pi_{0}^{*} \omega_{0}=-d \Theta_{(1,0)}
\end{aligned}
$$

As it is well known $\Omega_{(1,0)}$ is a symplectic form.

Let $i_{g}: \operatorname{Graph}(g) \hookrightarrow M_{0} \times M_{1}$ be the inclusion map, then

$$
i_{g}^{*} \Omega_{(1,0)}=\left(\pi_{0 \mid \operatorname{Graph}(g)}\right)^{*}\left(g^{*} \omega_{1}-\omega_{0}\right)
$$


Using this equality, it is clear that $g$ is a symplectomorphism if and only if $i_{g}^{*} \Omega_{(1,0)}=0$, that is, if $\operatorname{Graph}(g)$ is a Lagrangian submanifold of $\left(M_{0} \times M_{1}, \Omega_{(1,0)}\right)$.

Now, if $g$ is a symplectomorphism we have

$$
i_{g}^{*} \Omega_{(1,0)}=-d i_{g}^{*} \Theta_{(1,0)}=0
$$

and, therefore, at least locally, there exists a function $S: \operatorname{Graph}(g) \rightarrow \mathbb{R}$ such that

$$
i_{g}^{*} \Theta_{(1,0)}=d S
$$

Let $\left(q_{0}, p_{0}\right)$ and $\left(q_{1}, p_{1}\right)$ Darboux coordinates in $M_{0}$ and $M_{1}$, respectively. Since $\operatorname{Graph}(g)$ is diffeomorphic to $M_{0}$, we can take $\left(q_{0}, p_{0}\right)$ as natural coordinates in $\operatorname{Graph}(g)$. Since $\left(q_{0}, p_{0}, q_{1}, p_{1}\right)$ are coordinates in $M_{0} \times M_{1}$, then, along $\operatorname{Graph}(g)$, we have $q_{1}=q_{1}\left(q_{0}, p_{0}\right), p_{1}=p_{1}\left(q_{0}, p_{0}\right)$ and

$$
p_{1} d q_{1}-p_{0} d q_{0}=d S\left(q_{0}, p_{0}\right)
$$

\subsection{Generating functions of the first kind}

Assume that in a neighborhood of some point $x \in \operatorname{Graph}(g)$, we can change this system of coordinates by new independent coordinates $\left(q_{0}, q_{1}\right)$ (the local condition is that $\left.\operatorname{det}\left(\partial q_{1} / \partial p_{0}\right) \neq 0\right)$. In such case, the function $S$ can be expressed locally as $S=S\left(q_{0}, p_{0}\right)=S_{1}\left(q_{0}, q_{1}\right)$.

Definition 3.1 The function $S_{1}\left(q_{0}, q_{1}\right)$ will be called a generating function of the first kind of the symplectomorphism $g$.

From (11) we deduce that

$$
\left\{\begin{array}{l}
p_{0}=-\frac{\partial S_{1}}{\partial q_{0}} \\
p_{1}=\frac{\partial S_{1}}{\partial q_{1}}
\end{array}\right.
$$

(see Fig. 1).

Conversely, if $S_{1}\left(q_{0}, q_{1}\right)$ is a function such that $\operatorname{det}\left(\frac{\partial^{2} S_{1}}{\partial q_{0} \partial q_{1}}\right) \neq 0$ then $S_{1}\left(q_{0}, q_{1}\right)=$ $\left(p_{0}, p_{1}\right)$ is a generating function of some canonical transformation $g$ implicitly determined by Eqs. (12), $g\left(q_{0}, p_{0}\right)=\left(q_{1}, p_{1}\right)$ (see Arn:78).

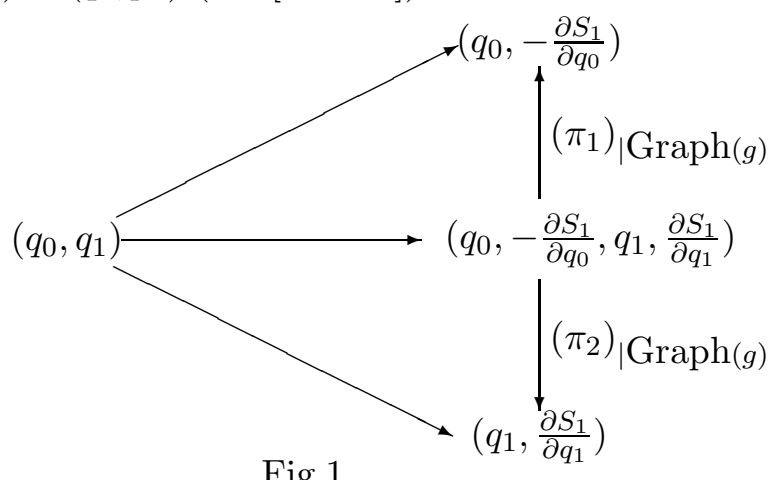

Fig.1 
Now suppose that $M$ is a fiber bundle over the real line $\mathbb{R}, \pi: M \rightarrow \mathbb{R}$, and $M_{t}=\pi^{-1}(t)$ are the fibers, where each fiber $M_{t}$ is equipped with a symplectic form $\omega_{t}$. Let $g_{(s, t)}: M_{t} \rightarrow M_{s}$ be a two-parameter family of symplectomorphisms satisfying

$$
g_{\left(t_{2}, t_{1}\right)} \circ g_{\left(t_{1}, t_{0}\right)}=g_{\left(t_{2}, t_{0}\right)}
$$

Next, we shall show how this composition law can be translated in terms of their respective generating functions. Moreover, the following results will give a geometric interpretation of the Discrete Euler-Lagrange equations [MarWes:01.

Theorem 3.2 Let $S_{1}^{\left(t_{N}, t_{0}\right)}$ be a function defined by

$$
S_{1}^{\left(t_{N}, t_{0}\right)}\left(q_{0}, q_{N}\right)=\sum_{k=0}^{N-1} S_{1}^{\left(t_{k+1}, t_{k}\right)}\left(q_{k}, q_{k+1}\right)
$$

where $q_{k} \in M_{t_{k}}, 1 \leq k \leq N-1$, are stationary points of the right-hand side, that is

$$
0=D_{2} S_{1}^{\left(t_{k}, t_{k-1}\right)}\left(q_{k-1}, q_{k}\right)+D_{1} S_{1}^{\left(t_{k+1}, t_{k}\right)}\left(q_{k}, q_{k+1}\right), \quad 1 \leq k \leq N-1 .
$$

If $S_{1}^{\left(t_{k}, t_{k-1}\right)}$ are generating functions of the first kind for $g_{\left(t_{k}, t_{k-1}\right)}$, then $S_{1}^{\left.t_{N}, t_{0}\right)}$ is a generating function of the first kind for $g_{\left(t_{N}, t_{0}\right)}: M_{t_{0}} \rightarrow M_{t_{N}}$.

Proof: Recursively, it is suffices to give the proof for $N=2$ :

$$
S_{1}^{\left(t_{2}, t_{0}\right)}\left(q_{0}, q_{2}\right)=S_{1}^{\left(t_{1}, t_{0}\right)}\left(q_{0}, x\right)+S_{1}^{\left(t_{2}, t_{1}\right)}\left(x, q_{2}\right)
$$

where $x$ is an stationary point of the right-hand side.

From the definitions of generating functions for $g_{\left(t_{2}, t_{1}\right)}$ and $g_{\left(t_{1}, t_{0}\right)}$

$$
\begin{aligned}
& p_{1} d q_{1}-p_{0} d q_{0}=d S^{\left(t_{1}, t_{0}\right)}\left(q_{0}, q_{1}\right) \\
& p_{2} d q_{2}-p_{1} d q_{1}=d S_{1}^{\left(t_{2}, t_{1}\right)}\left(q_{1}, q_{2}\right)
\end{aligned}
$$

and therefore

$$
p_{2} d q_{2}-p_{0} d q_{0}=d\left(S_{1}^{\left(t_{2}, t_{1}\right)}\left(q_{0}, q_{1}\right)+S_{1}^{\left(t_{1}, t_{0}\right)}\left(q_{1}, q_{2}\right)\right)
$$

It follows that

$$
0=D_{2} S_{1}^{\left(t_{1}, t_{0}\right)}\left(q_{0}, q_{1}\right)+D_{1} S_{1}^{\left(t_{2}, t_{1}\right)}\left(q_{1}, q_{2}\right)
$$

and, obviously, for this choice of $q_{1}$ then

$$
S_{1}^{h}\left(q_{0}, q_{1}\right)+S_{1}^{h}\left(q_{1}, q_{2}\right)
$$

is a generating function of the first kind of $g_{\left(t_{2}, t_{0}\right)}$.

Now, we are in condition to bring this procedure to the limit when the number of subintervals increases to infinity. Consider as its continuous counterpart a cosymplectic manifold $(M, \eta, \omega)$, where $M$ is still a fiber bundle over $\mathbb{R}\left(\pi_{\mathbb{R}}: M \rightarrow \mathbb{R}\right)$ and $\eta=$ 
$\pi_{\mathbb{R}}^{*}(d t)$. Denote by $M_{t}=\pi_{\mathbb{R}}^{-1}(t), t \in \mathbb{R}$. Take a Hamiltonian function $H: M \rightarrow \mathbb{R}$ and its Hamiltonian vector field $X_{H}$ given by

$$
i_{X_{H}} \omega=0 \quad \text { and } \quad i_{X_{H}} \eta=1
$$

Let $F_{(t, s)}: M_{s} \rightarrow M_{t}$ be the two-parameter family of symplectomorphisms generated by $X_{H}$ (see section 2) and consider as symplectic form on each fiber $M_{t}$ the restriction of $\omega$ to this fiber.

We shall give a characterization of the generating functions of the first kind associated to $F_{(t, s)}$ for $t$ close enough to $s$. For doing that, consider Darboux coordinates $\left(t, q^{A}, p_{A}\right)$ on $M$ and assume the regularity condition $\operatorname{det}\left(\frac{\partial^{2} H}{\partial p_{A} \partial p_{B}}\right) \neq 0$. Thus,

Proposition 3.3 A generating function of the first kind for $F_{(t, s)}$ is given by

$$
S_{1}^{\left(t_{1}, t_{0}\right)}\left(q_{0}, q_{1}\right)=\int_{t_{0}}^{t_{1}}(p(t) \dot{q}(t)-H(t, q(t), p(t))) d t
$$

where $t \rightarrow(t, q(t), p(t))$ is an integral curve of the Hamilton equations such that $q\left(t_{0}\right)=$ $q_{0}$ and $q\left(t_{1}\right)=q_{1}$.

Proof: We only use Hamilton equations and integration by parts:

$$
\begin{aligned}
\frac{\partial S_{1}^{\left(t_{1}, t_{0}\right)}}{\partial q_{0}}\left(q_{0}, q_{1}\right)= & \int_{t_{0}}^{t_{1}}\left(\frac{\partial p}{\partial q_{0}} \dot{q}+p \frac{\partial \dot{q}}{\partial q_{0}}-\frac{\partial H}{\partial q} \frac{\partial q}{\partial q_{0}}-\frac{\partial H}{\partial p} \frac{\partial p}{\partial q_{0}}\right) d t \\
= & \int_{t_{0}}^{t_{1}}\left(p \frac{\partial \dot{q}}{\partial q_{0}}+\dot{p} \frac{\partial q}{\partial q_{0}}\right) d t \\
& =-p_{0}+p_{1} \frac{\partial q_{1}}{\partial q_{0}}=-p_{0}
\end{aligned}
$$

and

$$
\begin{aligned}
\frac{\partial S_{1}^{\left(t_{1}, t_{0}\right)}}{\partial q_{1}}\left(q_{0}, q_{1}\right)= & \int_{t_{0}}^{t_{1}}\left(\frac{\partial p}{\partial q_{1}} \dot{q}+p \frac{\partial \dot{q}}{\partial q_{1}}-\frac{\partial H}{\partial q} \frac{\partial q}{\partial q_{1}}-\frac{\partial H}{\partial p} \frac{\partial p}{\partial q_{1}}\right) d t \\
= & \int_{t_{0}}^{t_{1}}\left(p \frac{\partial q}{\partial q_{1}}+\dot{p} \frac{\partial q}{\partial q_{1}}\right) d t \\
& =p_{1}-p_{0} \frac{\partial q_{0}}{\partial q_{1}}=p_{1}
\end{aligned}
$$

Remark 3.4 Suppose that $t_{i+1}-t_{i}=h$, for all $i=0, \cdots N-1$, then from Theorem 3.2 we have

$$
S_{1}^{N h}\left(q_{0}, q_{N}\right)=\sum_{k=0}^{N-1} S_{1}^{h}\left(q_{k}, q_{k+1}\right)
$$

where

$$
0=D_{2} S_{1}^{h}\left(q_{k-1}, q_{k}\right)+D_{1} S_{1}^{h}\left(q_{k}, q_{k+1}\right), \quad 1 \leq k \leq N-1 .
$$


Now, if we take as new generating function an adequate approximation $S_{d}^{h}$ of $S_{1}^{h}$ then

$$
0=D_{2} S_{d}^{h}\left(q_{k-1}, q_{k}\right)+D_{1} S_{d}^{h}\left(q_{k}, q_{k+1}\right), \quad 1 \leq k \leq N-1 .
$$

are the well-known Discrete Euler-Lagrange equations (see MarWes:01 and references therein). For instance, one can take

$$
S_{d}^{h}\left(q_{0}, q_{1}\right)=h \mathcal{L}\left(\alpha q_{0}+(1-\alpha) q_{1}, \frac{q_{1}-q_{0}}{h}\right), \quad \alpha \in[0,1]
$$

or alternatively, we could have considered more accurate approximations. Here, we are assuming that $\mathcal{L}: \mathbb{R} \times T Q \rightarrow \mathbb{R}$ is a Lagrangian function related via Legendre transformation with the Hamiltonian function $H$ (see Arn:78]) which is locally possible because of the regularity of $H$.

Denote by $S_{1}\left(q_{0}, q_{1}, t_{0}, t_{1}\right)=S_{1}^{\left(t_{1}, t_{0}\right)}\left(q_{0}, q_{1}\right)$. From Proposition (3.3), it is easy to show that:

$$
\begin{aligned}
& D_{3} S_{1}\left(q_{0}, q_{1}, t_{0}, t_{1}\right)=D_{3} S^{\left(t_{1}, t_{0}\right)}\left(q_{0}, q_{1}\right)=H\left(t_{0}, q_{0}, p_{0}\right) \\
& D_{4} S_{1}\left(q_{0}, q_{1}, t_{0}, t_{1}\right)=D_{4} S^{\left(t_{1}, t_{0}\right)}\left(q_{0}, q_{1}\right)=-H\left(t_{1}, q_{1}, p_{1}\right)
\end{aligned}
$$

(see also MarWes:01]). As a consequence

$$
D_{4} S^{\left(t_{k}, t_{k-1}\right)}\left(q_{k-1}, q_{k}\right)+D_{3} S^{\left(t_{k+1}, t_{k}\right)}\left(q_{k}, q_{k+1}\right)=0
$$

It should be noticed that if we take a new function $S_{d}^{\left(t_{k+1}, t_{k}\right)}$ as an adequate approximation of $S^{\left(t_{k+1}, t_{k}\right)}$, then solutions $\left\{q_{0}, q_{1}, \ldots, q_{N}\right\}$ of equations

$$
D_{2} S_{d}^{\left(t_{k}, t_{k-1}\right)}\left(q_{k-1}, q_{k}\right)+D_{1} S_{d}^{\left(t_{k+1}, t_{k}\right)}\left(q_{k}, q_{k+1}\right)=0, \quad 1 \leq k \leq N-1 .
$$

do not satisfy (13) for arbitrary values of $t_{k-1}, t_{k}, t_{k+1}$. Therefore, we may write the system of difference equations

$$
\left\{\begin{array}{l}
D_{2} S_{d}^{\left(t_{k}, t_{k-1}\right)}\left(q_{k-1}, q_{k}\right)+D_{1} S_{d}^{\left(t_{k+1}, t_{k}\right)}\left(q_{k}, q_{k+1}\right)=0, \\
D_{4} S_{d}^{\left(t_{k}, t_{k-1}\right)}\left(q_{k-1}, q_{k}\right)+D_{3} S_{d}^{\left(t_{k+1}, t_{k}\right)}\left(q_{k}, q_{k+1}\right)=0,
\end{array}\right.
$$

which under regularity assumptions will determine a time-dependent discrete flow

$$
\Phi\left(q_{k-1}, q_{k}, t_{k-1}, t_{k}\right)=\left(q_{k}, q_{k+1}, t_{k}, t_{k+1}\right)
$$

with variable step size $h_{k}=t_{k+1}-t_{k}$ (see KaMaOr:99, Lee:83, Lee:87, LeoMdD:2002, MarWes:01]).

\subsection{Generating functions of the second kind}

The construction of more general generating functions will be useful in next sections. For instance, suppose that $\left(q_{0}, p_{1}\right)$ are independent local coordinates on $\operatorname{Graph}(g)$. Then the function $S$ is written as $S=S\left(q_{0}, p_{1}\right)$.

We have

$$
p_{1} d q_{1}-p_{0} d q_{0}=-q_{1} d p_{1}+d\left(q_{1} p_{1}\right)-p_{0} d q_{0}=d S
$$

If we define

$$
S_{2}\left(q_{0}, p_{1}\right)=q_{1} p_{1}-S\left(q_{0}, p_{1}\right),
$$

where $q_{1}$ is expressed in terms of $q_{0}$ and $p_{1}$, then we deduce that

$$
q_{1} d p_{1}+p_{0} d q_{0}=d S_{2}\left(q_{0}, p_{1}\right)
$$


Definition 3.5 The function $S_{2}\left(q_{0}, p_{1}\right)$ will be called a generating function of the second kind of the symplectomorphism $g$.

We have that

$$
\left\{\begin{array}{l}
p_{0}=\frac{\partial S_{2}}{\partial q_{0}} \\
q_{1}=\frac{\partial S_{2}}{\partial p_{1}}
\end{array}\right.
$$

Conversely, if $S_{2}\left(q_{0}, p_{1}\right)$ is a generating function such that $\operatorname{det}\left(\frac{\partial^{2} S_{2}}{\partial q_{0} \partial p_{1}}\right) \neq 0$ then $S_{2}$ is a generating function of some local symplectomorphism determined by Eqs. (15) (see Arn:78]).

Denote by $F_{\left(t_{2}, t_{1}\right)}: M_{t_{1}} \rightarrow M_{t_{2}}$ the two-parametric group of canonical transformations generated by the Hamiltonian vector field $X_{H}$, as in the preliminaries to Proposition 3.3. We have the following.

Theorem 3.6 Let a function $S_{2}^{\left(t_{N}, t_{0}\right)}$ be defined by

$$
S_{2}^{\left(t_{N}, t_{0}\right)}\left(q_{0}, p_{N}\right)=\sum_{k=0}^{N-1} S_{2}^{\left(t_{k+1}, t_{k}\right)}\left(q_{k}, p_{k+1}\right)-\sum_{k=1}^{N-1} q_{k} p_{k}
$$

where $q_{k}, 1 \leq k \leq N$, and $p_{k}, 0 \leq k \leq N-1$, are stationary points of the right-hand side, that is

$$
\begin{array}{ll}
q_{k}=\frac{\partial S_{2}^{\left(t_{k-1}, t_{k}\right)}}{\partial p}\left(q_{k-1}, p_{k}\right), \quad 1 \leq k \leq N, \\
p_{k}=\frac{\partial S_{2}^{\left(t_{k}, t_{k+1}\right)}}{\partial q}\left(q_{k}, p_{k+1}\right), \quad 0 \leq k \leq N-1,
\end{array}
$$

then $S_{2}^{\left(t_{N}, t_{0}\right)}$ is a generating function of the second kind for $F_{\left(t_{N}, t_{0}\right)}: M_{t_{0}} \rightarrow M_{t_{N}}$.

Proof: It follows as in Theorem 3.2

As a consequence, we have that

$$
S^{\left(t_{N}, t_{0}\right)}\left(q_{0}, p_{N}\right)=q_{N} p_{N}-S_{2}^{\left(t_{N}, t_{0}\right)}\left(q_{0}, p_{N}\right)=\sum_{k=0}^{N-1}\left[q_{k+1} p_{k+1}-S_{2}^{\left(t_{k+1}, t_{k}\right)}\left(q_{k}, p_{k+1}\right)\right]
$$

where the unknown coordinates are given by (17) and (18).

Proposition 3.7 A generating function of the second kind for $F_{\left(t_{1}, t_{0}\right)}$ is given by

$$
S_{2}^{\left(t_{1}, t_{0}\right)}\left(q_{0}, p_{1}\right)=p_{1} q_{1}-\int_{t_{0}}^{t_{1}}(p(t) \dot{q}(t)-H(t, q(t), p(t))) d t
$$

where $\rightarrow(q(t), p(t))$ is an integral curve of the Hamilton equations such that $q\left(t_{0}\right)=q_{0}$ and $p\left(t_{1}\right)=p_{1}$. 
Proof: It is proved in a similar way to Proposition 3.3 ,

Denote by $S_{2}\left(t, q_{0}, p_{1}\right)=S_{2}^{(0, t)}\left(q_{0}, p_{1}\right)$ then it is easy to show that (see, for instance HaLuWa:02 )

Theorem 3.8 (Hamilton-Jacobi equation for $S_{2}$ ) If $S_{2}\left(t, q_{0}, p_{1}\right)$ is a solution of the partial differential equation

$$
\frac{\partial S_{2}}{\partial t}=H\left(\frac{\partial S_{2}}{\partial p_{1}}\left(t, q_{0}, p_{1}\right), p_{1}\right), \quad S_{2}\left(0, q_{0}, p_{1}\right)=q_{0} p_{1}
$$

then the mapping $\left(q_{0}, p_{0}\right) \longrightarrow\left(q_{1}, p_{1}\right)$ defined by Eqs. (15) is the exact flow of the Hamiltonian system determined by $H$.

\section{Optimal control of Discrete-time systems}

In this section we shall define the general solution of an optimization problem for discrete systems and analyze its geometric behaviour, in particular, the symplecticity.

Suppose that the discrete state equations are given by the dynamical equation

$$
q_{k+1}^{A}=f^{A}\left(k, q_{k}, u_{k}\right), \quad k=0,1, \ldots, N-1, \quad A=1,2, \ldots, m
$$

or, shortly, $q_{k+1}=f\left(k, q_{k}, u_{k}\right)$, where $q_{0}$ is initially given.

The associate performance index or objective function is:

$$
J=\bar{S}(N, q(N))+\sum_{k=0}^{N-1} \bar{L}\left(k, q_{k}, u_{k}\right)
$$

where $\bar{S}$ is a function of the final time and state at the final time $N$, and $\bar{L}$ is timevarying function of the state and control input at each intermediate discrete time $k$.

The optimal control problem is solved finding controls $u_{k}^{*}, k=0,1, \ldots N-1$, that drive the system along a trajectory $q_{k}^{*}, k=0,1, \ldots, N$, verifying the state equations such that the performance index is minimized.

\subsection{Problem solution}

Let us now solve the optimal control problem for the discrete optimal problem determined by (21) and (22) using the Lagrange multiplier approach. Considering the state Eqs. (21) as constraint equations, then we have $N \cdot m$ constraints, and we associate a Lagrange multiplier to each constraint. Next, we construct the augmented performance index $J^{\prime}$ by

$$
J^{\prime}=\sum_{k=0}^{N-1}\left[p_{k+1}\left(f\left(k, q_{k}, u_{k}\right)-q_{k+1}\right)-\bar{L}\left(k, q_{k}, u_{k}\right)\right]-\bar{S}(N, q(N))
$$

where $p_{k+1}=\left(\left(p_{k+1}\right)_{A}\right)$ are considered as Lagrange multipliers with $A=1, \ldots, n$ and $k=0, \ldots, N-1$.

Taking the Hamiltonian function

$$
\bar{H}\left(k, q_{k}, p_{k+1}, u_{k}\right)=p_{k+1} f\left(k, q_{k}, u_{k}\right)-\bar{L}\left(k, q_{k}, u_{k}\right)
$$


we deduce that the necessary conditions for a constrained minimum are thus given by:

$$
\begin{aligned}
q_{k+1} & =\frac{\partial \bar{H}}{\partial p}\left(k, q_{k}, p_{k+1}, u_{k}\right)=f\left(k, q_{k}, u_{k}\right) \\
p_{k} & =\frac{\partial \bar{H}}{\partial q}\left(k, q_{k}, p_{k+1}, u_{k}\right)=p_{k+1} \frac{\partial f}{\partial q}\left(k, q_{k}, u_{k}\right)-\frac{\partial \bar{L}}{\partial q}\left(k, q_{k}, u_{k}\right) \\
0 & =\frac{\partial \bar{H}}{\partial u}\left(k, q_{k}, p_{k+1}, u_{k}\right)=p_{k+1} \frac{\partial f}{\partial u}\left(k, q_{k}, u_{k}\right)-\frac{\partial \bar{L}}{\partial u}\left(k, q_{k}, u_{k}\right)
\end{aligned}
$$

where $0 \leq k \leq N-1$, and the transversality conditions

$$
p_{N}=-\frac{\partial \bar{S}}{\partial q}\left(N, q_{N}\right) \quad \text { and } \quad q_{0} \quad \text { fixed. }
$$

Observe that the recursion for the state $q_{k}$ develops forward in time, but the co-state variable $p_{k}$ develops backwards in time. Therefore the required boundary conditions for finding a solution are the initial state $q_{0}$ and the final co-state $p_{N}$.

Assume that

$$
\operatorname{det}\left(\frac{\partial^{2} \bar{H}}{\partial u_{a} \partial u_{b}}\right) \neq 0
$$

then, locally, $u_{k}^{*}=h\left(k, q_{k}, p_{k+1}\right)$. If we denote, by

$$
\tilde{H}\left(k, q_{k}, p_{k+1}\right)=\bar{H}\left(k, q_{k}, p_{k+1}, u_{k}^{*}\right)
$$

then Eqs. (24), (25) are rewritten as

$$
\begin{aligned}
q_{k+1} & =\frac{\partial \tilde{H}}{\partial p}\left(k, q_{k}, p_{k+1}\right) \\
p_{k} & =\frac{\partial \tilde{H}}{\partial q}\left(k, q_{k}, p_{k+1}\right)
\end{aligned}
$$

with $0 \leq k \leq N_{1}$.

Consider the function

$$
G_{k}\left(q_{k}, q_{k+1}, p_{k+1}\right)=\tilde{H}\left(k, q_{k}, p_{k+1}\right)-p_{k+1} q_{k+1}, \quad 0 \leq k \leq N-1 .
$$

Then, for a fixed $k$ :

$$
d G_{k}=\frac{\partial \bar{H}}{\partial q_{k}}\left(k, q_{k}, p_{k+1}\right) d q_{k}+\frac{\partial \bar{H}}{\partial p_{k+1}}\left(k, q_{k}, p_{k+1}\right) d p_{k+1}-p_{k+1} d q_{k+1}-q_{k+1} d p_{k+1} .
$$

Along solutions of Eqs. (24), (25) and (26) we have:

$$
d G_{k \mid \mathrm{Sol}}=p_{k} d q_{k}-p_{k+1} d q_{k+1},
$$

which implies

$$
d p_{k} \wedge d q_{k}=d p_{k+1} \wedge d q_{k+1} .
$$

along the solution of (24)-(26).

In the next subsection, we shall analyze the geometric meaning of Eq. (29), which it is obviously interpreted as symplecticity of discrete optimal control problems in terms of a natural symplectic form. 


\subsection{Generating functions of the second kind and discrete optimal control problems}

From Proposition 3.3 the following function is a generating function of the second kind for the cosymplectic Hamiltonian system $\left(P_{0}, \eta, \Omega, H_{\mid P_{0}}\right)$, which determines the dynamics of the optimal control problem given by (11) and (2):

$$
S_{2}^{\left(t_{1}, t_{0}\right)}\left(q_{0}, p_{1}\right)=p_{1} q_{1}-\int_{t_{0}}^{t_{1}}\left(p(t) \dot{q}(t)-H_{\mid P_{0}}(t, q(t), p(t))\right) d t
$$

where $t \rightarrow(t, q(t), p(t))$ is the integral curve on $P_{0}$ of the vector field $X_{P_{0}}$. Here $X_{P_{0}}$ is the unique solution of equation

$$
i_{X_{P_{0}}} \Omega=d H_{\mid P_{0}} \quad i_{X_{P_{0}}} \eta=1
$$

with $\left(q\left(t_{0}\right), p\left(t_{0}\right)\right)=\left(q_{0}, p_{0}\right)$ and $\left(q\left(t_{1}\right), p\left(t_{1}\right)\right)=\left(q_{1}, p_{1}\right)$.

We now focus on the construction of a numerical integrator for the Hamiltonian system $\left(P_{0}, \eta, \Omega, H_{\mid P_{0}}\right)$ by using an approximation of the generating function. As we shall show, the obtained method also realize the integration steps by symplectomorphism transformations; then, it is a symplectic integrator.

First take a fixed time interval $h=t_{k+1}-t_{k}, k=0, \ldots, N-1$.

Assume that we are working on vector spaces, and consider the following natural approximation:

$$
\begin{aligned}
\tilde{S}_{2}^{h}\left(k, q_{k}, p_{k+1}\right)= & p_{k+1} q_{k+1}-h p_{k+1}\left(\frac{q_{k+1}-q_{k}}{h}\right)-h \tilde{L}\left(k, q_{k}, p_{k+1}\right) \\
& +h p_{k+1} \tilde{\Gamma}\left(k, q_{k}, p_{k+1}\right)
\end{aligned}
$$

where, for instance, $\tilde{L}\left(k, q_{k}, p_{k+1}\right)=L_{\mid P_{0}}\left(t_{0}+k h, q_{k}, p_{k+1}\right)$ and $\tilde{\Gamma}\left(k, q_{k}, p_{k+1}\right)=\Gamma_{\mid P_{0}}\left(t_{0}+\right.$ $\left.k h, q_{k}, p_{k+1}\right)$.

If we denote by $\tilde{f}\left(k, q_{k}, p_{k+1}\right)$ the function

$$
\tilde{f}\left(k, q_{k}, p_{k+1}\right)=h \tilde{\Gamma}\left(k, q_{k}, p_{k+1}\right)+q_{k}
$$

then,

$$
\tilde{S}_{2}^{h}\left(k, q_{k}, p_{k+1}\right)=p_{k+1} \tilde{f}\left(k, q_{k}, p_{k+1}\right)-\tilde{L}\left(k, q_{k}, p_{k+1}\right)=\tilde{H}\left(k, q_{k}, p_{k+1}\right) .
$$

Thus, equations

$$
\left\{\begin{array}{l}
p_{k}=\frac{\partial \tilde{S}_{2}^{h}}{\partial q^{k}}\left(k, q_{k}, p_{k+1}\right)=\frac{\partial \tilde{H}}{\partial q^{k}}\left(k, q_{k}, p_{k+1}\right) \\
q_{k+1}=\frac{\partial \tilde{S}_{2}^{h}}{\partial p_{k+1}}\left(k, q_{k}, p_{k+1}\right)=\frac{\partial \tilde{H}}{\partial p_{k+1}}\left(k, q_{k}, p_{k+1}\right)
\end{array}\right.
$$

are exactly (27) and (28) and the symplecticity condition (29) for discrete optimal control problems is now a trivial consequence of the generating function construction.

Remark 4.1 It is also possible to construct symplectic numerical methods of higher order; for instance, considering better approximations of the Hamilton Jacobi equation 
(20) (see [ChaSco:90 and references therein). Assume for simplicity that the Hamiltonian is autonomous, that is, $H \equiv H(q, p)$. Now, first expands the generating function $S_{2}\left(t, q_{0}, p_{1}\right)$ as:

$$
S_{2}\left(t, q_{0}, p_{1}\right)=q_{0} p_{1}+\sum_{i=1}^{\infty} t^{i} G_{i}\left(q_{0}, p_{1}\right),
$$

inserts expression into Hamilton-Jacobi equation (20) and compares equal powers of $t$. This yields

$$
\begin{aligned}
G_{1}\left(q_{0}, p_{1}\right) & =H\left(q_{0}, p_{1}\right) \\
G_{2}\left(q_{0}, p_{1}\right) & =\frac{1}{2}\left(\frac{\partial H}{\partial q_{0}^{A}} \frac{\partial H}{\partial p_{1 A}}\right) \\
G_{3}\left(q_{0}, p_{1}\right) & =\frac{1}{6}\left(\frac{\partial^{2} H}{\partial p_{1 A} \partial p_{1 B}} \frac{\partial H}{\partial q_{0}^{A}} \frac{\partial H}{\partial q_{0}^{B}}+\frac{\partial^{2} H}{\partial p_{1 A} \partial q_{0}^{B}} \frac{\partial H}{\partial q_{0}^{A}} \frac{\partial H}{\partial p_{1 B}}+\frac{\partial^{2} H}{\partial q_{0}^{A} \partial q_{0}^{B}} \frac{\partial H}{\partial p_{1 A}} \frac{\partial H}{\partial p_{1 B}}\right) \\
\ldots & =\ldots
\end{aligned}
$$

Using the truncated series, we obtain an approximated generating function:

$$
S_{2}^{h}\left(q_{k}, p_{k+1}\right)=q_{k} \cdot p_{k+1}+\sum_{i=1}^{r} h^{r} G_{i}\left(q_{k}, p_{k+1}\right)
$$

which defines a symplectic method of order $r$.

Other approaches are also admissible without using higher derivatives of the Hamiltonian $H$, for instance, symplectic or symplectic partitioned Runge-Kutta methods (see HaLuWa:02, SanCal:94).

\section{Discrete Hamiltonian systems}

In ErbYan:92] Erbe and Yan have considered discrete linear Hamiltonian systems of the form:

$$
\begin{aligned}
& \Delta y(t)=B(t) y(t+1)+C(t) z(t) \\
& \Delta z(t)=-A(t) y(t+1)-B^{T}(t) z(t)
\end{aligned}
$$

where $A, C$ are symmetric and $I-B$ is invertible. Here $\Delta y(t)=y(t+1)-y(t)$, $\Delta z(t)=z(t+1)-z(t)$ and $y, z \in \mathbb{R}^{d}$.

This problem is a particular case of a discrete Hamiltonian systems of the form

$$
\begin{aligned}
& \Delta y(t)=H_{z}(t, y(t+1), z(t)) \\
& \Delta z(t)=-H_{y}(t, y(t+1), z(t))
\end{aligned}
$$

where $H(t, y, z)=\frac{1}{2}\left(y^{T}, z^{T}\right)\left(\begin{array}{cc}A(t) & B^{T}(t) \\ -B(t) & C(t)\end{array}\right)\left(\begin{array}{c}y \\ z\end{array}\right)$. The symplecticity of the discrete linear Hamiltonian system was fully studied (see ErbYan:92, for instance, and references therein). The existence of a corresponding symplectic structure for discrete nonlinear Hamiltonian systems given by (33) and (34) was proposed by Ahlbrandt as an open problem ([Ahlb:93] and also Shi:02]). 
From the point of view of section 3, this open problem is easily solved considering as generating function of the second kind the following one:

$$
S_{2}^{(t+1, t)}(y(t+1), z(t))=z(t) y(t+1)-H(t, y(t+1), z(t)) .
$$

Then Eqs. (33) and (34) are precisely

$$
\left\{\begin{aligned}
y(t) & =\frac{\left.\partial S^{(t+1, t}\right)}{\partial z}(y(t+1), z(t)) \\
z(t+1) & =\frac{\partial S^{(t+1, t)}}{\partial y}(y(t+1), z(t)),
\end{aligned}\right.
$$

which guarantees the symplecticity of the discrete Hamiltonian system. In order to find the canonical transformation associated to this generating map it is only necessary to impose the local condition (see [Arn:78]):

$$
\operatorname{det}\left(\frac{\partial^{2} S_{2}^{(t+1, t)}(y(t+1), z(t)}{\partial y \partial z}\right) \neq 0
$$

Then, in a neighbourhood of a point satisfying the above condition, there exists a symplectomorphism defined by Eqs. (33) and (34).

\section{Acknowledgments}

This work has been supported by grant BFM2001-2272 (Ministry of Science and Technology, Spain). A. Santamaría Merino wishes to thank the Programa de Formación de Investigadores of the Departamento de Educación, Universidades e Investigación of the Basque Government (Spain) for financial support.

\section{References}

[Ahlb:93] Ahlbrandt C D 1993 Equivalence of Discrete Euler Equations and Discrete Hamiltonian Systems, J. Math. Anal. Appl. 180, 498-478

[Arn:78] Arnold V I 1978 Mathematical Methods of Classical Mechanics (Graduate Text in Mathematics 60, Springer-Verlag New York)

[BobSus:99a] Bobenko A I and Y B Suris 1999 Discrete Lagrangian reduction, discrete Euler-Poincaré equations, and semidirect products Lett. Math. Phys. 49, 79-93

[BobSus:99b] Bobenko A I and Suris Y B 1999 Discrete time Lagrangian mechanics on Lie groups, with an application to the Lagrange top Comm. Math. Phys. 204 $147-188$

[Cadz:70] Cadzow J A 1970 Discrete calculus of variations Intern. J. Control. 11, 393-407

[ChaSco:90] Channell P J and Scovel C 1990 Symplectic integration of Hamiltonian Systems, Nonlinearity 3, 231-259

[ChLeMa:94] Chinea D, de León M and Marrero J C 1994 The constraint algorithm for time-dependent Lagrangians, J. Math. Phys. 35 (7), 3410-3447

[Cort:02] Cortés J 2002 Geometric, control and numerical aspects of nonholonomic systems (Lecture Notes in Mathematics, vol. 1793, Springer-Verlag) 
[CorMar:01] Cortés J and Martínez S 2001 Nonholonomic integrators Nonlinearity 14, $1365-1392$

[Dirac:64] Dirac P A M 1964 Lecture on Quantum Mechanics (Belfer Graduate School of Science, Yeshiva University, New York)

[ErbYan:92] Erbe L H and Yan P 1992 Disconjugancy for linear Hamiltonian difference systems, J. Math. Anal. Appl. 167, 355-367

[GotNes:79] Gotay M J and Nester J M 1979 Presymplectic Lagrangian systems I: The Constraint Algorithm and the Equivalence Theorem, Ann. Inst. Henri Poincaré, A30 129-142

[HaLuWa:02] Hairer E Lubich C and Wanner G 2002 Geometric Numerical Integration, Structure-Preserving Algorithms for Ordinary Differential Equations (Springer Series in Computational Mathematics 31, Springer-Verlag Berlin Heidelberg)

[JarNor:97a] Jaroszkiewicz G and Norton K 1997 Principes of discrete time mechanics I: Particle systems J. Phys. A 30, 3115-3144

[JarNor:97b] Jaroszkiewicz G and Norton K 1997 Principes of discrete time mechanics II: Classical field theory J. Phys. A 30, 3145-3163

[JorPol:64] Jordan B W and Polak E 1964 Theory of a class of discrete optimal control systems J. Electron. Control 17, 697-711

[KaMaOr:99] Kane C Marsden J E and Ortiz M 1999 Symplectic energy-momentum integrators J. Math. Phys. 40, 3353-3371

[KMOW:00] Kane C Marsden J E Ortiz M and West M 2000 Variational integrators and the Newmark algorithm for conservative and dissipative mechanical systems Internat. J. Numer. Math. Eng. 49, 1295-1325.

[Lee:83] Lee T D 1983 Can time be a discrete dynamical variable? Phys. Lett., 122B, $217-220$

[Lee:87] Lee T D 1987 Difference equations and conservation laws J. Statis. Phys., 46, 843-860

[LeMaMD:96] de León M Marrero J C and Martín de Diego D 1996 Time-dependent constrained Hamiltonian systems and Dirac brackets Journal of Physics A: Math. Gen. 29 6843-6859

[LeMDSa:02a] de León M Martín de Diego D and Santamaría A 2002 Geometric integrators and nonholonomic mechanics, Preprint IMAFF-CSIC

[LeMDSa:02b] de León M, Martín de Diego D and Santamaría A 2003 Geometric numerical integration of nonholonomic systems and optimal control problems 2nd IFAC Workshop on lagrangian and Hamiltonian Methods for Nonlinear Control, Seville 2003, 163-168.

[LeoMdD:2002] de León M and Martín de Diego D 2002 Variational integrators and time-dependent Lagrangian systems Rep. on Math. Phys 49 2/3, 183-192

[Lew:86] Lewis F.L 1986 Optimal Control (John Wiley\& Sons, New York)

[Mae:80] Maeda S 1980 Canonical structure and symmetries for discrete systems Math. Japonica 25, 405-420 
[Mae:81] Maeda S 1981 Extension of discrete Noether theorem Math. Japonica 26, 85-90

[MaPaSh:98] Marsden J E Patrick G W and Shkoller S 1998 Multisymplectic geometry, variational integrators, and nonlinear PDEs' Comm. Math. Phys. 199, 351- 395

[MarWes:01] Marsden J E and West M 2001 Discrete mechanics and variational integrators Acta Numerica, 357-514

[MosVes:91] Moser J and Veselov A P 1991 Discrete versions of some classical integrable systems and factorization of matrix polynomials Comm. Math. Phys. 139, 217-243

[Nijvan:90] Nijmeijer H and van der Schaft A J 1990 Nonlinear dynamical control systems (Springer-Verlag, New York)

[NorJar:98] Norton K and Jaroszkiewicz G 1998 Principes of discrete time mechanics, III: Quantum field theory J. Phys. A 31, 977-1000

[PKMO:02] Pandolfi A Kane C Marsden J E and Ortiz M 2002 Time-discretized variational formulation of nonsmooth frictional contact Int. J. Num. Methods in Engineering 53, 1801-1829

[SanCal:94] Sanz-Serna J M and Calvo M P 1994 Numerical Hamiltonian Problems (Chapman\& Hall, London)

[Shi:02] Shi Y 2002 Symplectic structure of Discrete Hamiltonian Systems, J. Math. Anal. Appl. 266, 472-478 